\title{
Sigma-1 receptor activation ameliorates anxiety-like behavior through NR2A-CREB-BDNF signaling pathway in a rat model submitted to single-prolonged stress
}

\author{
LI-LI JI, JUN-BO PENG, CHANG-HAI FU, LEI TONG and ZHEN-YU WANG
}

Department of Anatomy, College of Basic Medical Sciences, China Medical University, Shenyang, Liaoning 110122, P.R. China

Received January 4, 2017; Accepted July 11, 2017

DOI: $10.3892 / \mathrm{mmr} .2017 .7185$

\begin{abstract}
Accumulating evidence has demonstrated that the $\sigma-1$ receptor $(\sigma-1 R)$ possesses neuroprotective effects and is a potential novel therapeutic target forcertain psychiatric diseases, including post-traumatic stress disorder (PTSD) accompanied with anxiety disorder. It has been reported that $\sigma-1 \mathrm{R}$ agonist treatment could be modulated by the brain-derived neurotrophic factor (BDNF) signaling pathway. However, it remains unclear whether BDNF and its upstream regulator are mechanistically involved in the therapeutic effect of $\sigma-1 \mathrm{R}$ in PTSD. To address this question, rats were subjected to a single-prolonged stress (SPS) paradigm and $\sigma-1 \mathrm{R}$ agonist administration. Open-field and elevated plus maze tests were implemented to evaluate the effect of $\sigma-1 \mathrm{R}$ activation on the improvement of anxiety-like behaviors. Furthermore, the expression levels of BDNF, phosphorylated cAMP responsive element-binding protein (CREB) and glutamate receptor ionotropic $\mathrm{N}$-methyl $\mathrm{D}$-aspartate $2 \mathrm{~A}$ (NMDAR2A) were investigated in the hippocampi of rats. It was revealed that the downregulation of BDNF, phosphorylated CREB and NMDAR2A induced by SPS were reversed by $\sigma-1 R$ activation. Collectively, the results of the present study suggest that the NMDAR2A/CREB/BDNF signaling pathway is involved in the activation of $\sigma-1 \mathrm{R}$ resulting in therapeutic effects for PTSD.
\end{abstract}

\section{Introduction}

Post-traumatic stress disorder (PTSD) is an anxiety disorder induced by undergoing life-threatening events, such as natural disasters, combat or violent assaults (1). It is the fourth most common psychiatric diagnosis and classified as anxiety

Correspondence to: Professor Lei Tong or Professor Zhen-Yu Wang, Department of Anatomy, College of Basic Medical Sciences, China Medical University, 77 Puhe Road, Shenyang, Liaoning 110122, P.R. China

E-mail: tonglei@cmu.edu.cn

E-mail: zywang@live.cn

Key words: sigma-1 receptor, PRE084, post-traumatic stress disorder, brain-derived neurotrophic factor disorder. One of the characteristic features of PTSD patients is avoidance of the traumatic-related stimuli persistently. But the neurocircuitry mechanism underlining PTSD is not fully understood now. It has been identified that three areas of the brain whose function may be involved in PTSD: the prefrontal cortex, amygdala and hippocampus, among which the hippocampus is a limbic system structure involved in memory process, as well as being a regulatory center for stress response (2-4). Some studies suggested that the volume of hippocampus reduced in patients with major PTSD disorder (5-7).

It has been reported that sigma-1 receptor (Sig-1R), a transmembrane protein located in the endoplasmic reticulum (ER) membrane, is highly expressed in brain tissue $(8,9)$. It regulates lipid transport, synaptic signaling, axonal out ground, the activity of various ion channels and neurogenesis in nervous system (10-14). Sig-1Rs are well known as the therapeutic targets for antidepressant drugs because of their antidepressant-like effects (15). Sig-1R knock-down in vitro can lead to calcium homeostasis defects and stress activation, and thus enhance apoptosis induced by stress factor $(16,17)$. But the possible effects of Sig-1R on animal model of PTSD have not yet been reported. A report suggested that the brain-derived neurotrophic factor (BDNF) signaling pathway could be modulated through the Sig-1R chaperone by chronic treatment with antidepressants (15). Our previous study also found that BDNF-TrkB (Tropomyosin receptor kinase B, TrkB)-ERK (extracellular regulated protein kinases, ERK) signaling pathway was involved in the neuroprotective effect in reduction of anxiety-like behaviors by Sig-1R activation in PTSD rats (18). But whether BDNF affects other pathways is unclear. BDNF, a neurotrophic released during high-frequency stimulation patterns, plays a critical role in the survival, differentiation and synaptic plasticity of mammalian neurons. It has been shown that BDNF can regulate the phosphorylation, trafficking, and expression of N-methyl-D-aspartate (NMDA) receptor, a heteromeric complexes composed of two glycine-binding NR1 subunits in combination with various glutamate binding NR2 subunits (NR2A, NR2B, NR2C and NR2D) (19). Previous study has proposed that NMDAR blockade may be beneficial for anxiety patients presented with impaired extinction responses, particularly after repeated unpredictable traumatic experiences (20). Among subunits of NMDAR, NR2A is predominantly located at synapses and 
the activation of NR2A promotes neuronal survival $(21,22)$. cAMP responsive element-binding protein (CREB), whose phosphorylation can regulate the action of BDNF in cells, is associated with synaptic NMDARs and may mediate their pro-survival effects $(23,24)$. It has been shown that the lower level of p-CREB in long-term at standard condition (LTSC) mice and its behavior could be associated with the development of anxiety (25).

In the present study, we evaluate the anti-anxiety effects of Sig-1R on single-prolonged stress (SPS) rats, which has been extensively developed and employed in the investigation of PTSD. Rats are chronically treated with a Sig-1R high-selective agonist 2-morpholin-4-ylethyl 1-phenylcyclohexane-carboxylate (PRE-084) to activate Sig-1R. Then we use open-field test (OFT) and elevated plus maze test (EPM) to examine anxiety-like behavior of SPS rats. We analyzed BDNF, phosphorylated CREB (p-CREB) and NR2A expressions in the hippocampi of rats to clarify possible mechanisms of anti-anxiety effect by Sig-1R activation in PTSD animal models.

\section{Materials and methods}

Animals. All experimental procedures on animals were approved by the Institutional Animal Care and Use Committee (China Medical University) and were in accordance with the National Institutes of Health Guide for the care and use of laboratory animals. Male Sprague-Dawley rats (Approval number: SCXK (liao 2015-0001), approximately $200 \mathrm{~g}$ weight, 3-4 months of age) were obtained from the Experimental Animal Center of China Medical University. Animals were kept at constant temperature $22^{\circ} \mathrm{C}$, humidity at 40-60\% and under a 12-h light/dark cycle with food and water freely available. Following an adaptation period for at least one week, the experimental procedures were undertaken.

Experimental groups and SPS model. Forty-eight rats were randomly divided into four groups: Sham + Vehicle: normal rats treated with physiological saline; Sham + PRE-084: normal rats treated with PRE-084; SPS + Vehicle: SPS rats treated with physiological saline; SPS + PRE-084: SPS rats treated with PRE-084; Behavioral tests were carried out with all the rats and we chose the results of 6 rats randomly to do the statistical analysis. 6 rats of each group were used for immunofluorescence staining and the other 6 rats were used for western blot.

The SPS model was built as previously described with slight modifications $(26,27)$. Briefly, rats were restrained inside a disposable restraint holder (58 $\mathrm{mm}$ diameter, $150 \mathrm{~mm}$ length) for $2 \mathrm{~h}$. After that, they were put in a clear plastic container $(600 \times 400 \times 500 \mathrm{~mm})$ filled two thirds with water $\left(24^{\circ} \mathrm{C}\right)$ and forced to swim for $20 \mathrm{~min}$ individually. Then animals were permitted to rest for $15 \mathrm{~min}$. Finally, rats were exposed to diethyl ether until loss of consciousness.

Drug infusion. PRE-084 (Sigma-Aldrich, St. Louis, MO, USA) was dissolved in sterile saline before use. After the SPS procedure, PRE-084 or saline was infused at the volume of $0.1 \mathrm{ml} / 10 \mathrm{~g}$ body weight by intraperitoneal injections daily for 7 consecutive days. The dose of PRE-084 was $0.6 \mathrm{mg} / \mathrm{kg}$ based on the previous reports (18).

\section{Behavioral tests}

Open-field test $(O F T)$. Locomotor, exploratory and anxiety-like behaviors were detected by open-field test. The apparatus was a black Plexiglas enclosure measuring 50x50x50 cm with a red fluorescent light illumination over the center of the arena. In present study, rats were subjected with OFT after drug treatment. Briefly, after $30 \mathrm{~min}$ of acclimation in the room, rats were placed at a central start position in the open arena and allowed to explore for $5 \mathrm{~min}$. After each session, the arena was cleaned by $70 \%$ ethanol and each rat was tested only once.

Elevated plus maze test. The elevated plus maze (EPM) test was performed after OFT as previously described (28). Briefly, the EPM apparatus is made up of two opposing open and two opposing closed arms $(50 \mathrm{~cm}$ arms, $50 \mathrm{~cm}$ off the ground). Rats were put on the central platform facing an open arm and allowed to explore for $5 \mathrm{~min}$. Each test was videotaped and scored by an independent observer subsequently. Arm entry was defined as entering an arm with all four paws. Anxiety behavior was quantified by the percentage of time spent in the open arms and percentage of entries into the open arms relative to total (open + closed) arm.

Fixation and sections preparation of the hippocampus. After behavioral tests, six rats of each group were anesthetized with pentobarbital sodium $(30 \mathrm{mg} / \mathrm{kg}$ i.p.) and then were perfused from left ventricle with $300 \mathrm{ml} 4 \%$ cold paraformaldehyde in phosphate-buffered saline (PBS, pH7.4). The whole brain was removed rapidly and post-fixed for $10 \mathrm{~h}$ in $4 \%$ cold paraformaldehyde in phosphate-buffered saline at $4^{\circ} \mathrm{C}$. Then the brain was immersed in $30 \%$ sucrose solution for $24 \mathrm{~h}$ and sliced into $7 \mu \mathrm{m}$ coronal sections. The sections were stored at $-70^{\circ} \mathrm{C}$ before use.

Immunofluorescence staining. The sections were permeabilized by $0.3 \%$ Triton X-100 in PBS for $30 \mathrm{~min}$ at room temperature. After being washed 3 times with PBS, the sections were treated with blocking solution (5\% bovine serum albumin in PBS) for $20 \mathrm{~min}$. Then the sections were incubated with mouse monoclonal antibody against NeuN (Abcam, Cambridge, MA, USA, 1:200) overnight at $4^{\circ} \mathrm{C}$. After being washed with PBS, the sections were incubated with Alexa Fluor 594 IgG secondary antibodies (Thermo Fisher Scientific, MA, USA; 1:400) for $1 \mathrm{~h}$ at $37^{\circ} \mathrm{C}$. A few sections in every experiment were incubated in PBS without primary antibody to assess nonspecific staining.

Six slides were selected from each group randomly, and six visual fields were randomly selected on each slide. The average value was evaluated by the optical density (OD) of positive cells in each field, and then the OD of the NeuN-immunopositive cells was analyzed through MetaMorph/DPIO/BX41 morphology image analysis system.

Western blot analysis. The hippocampi of the other six rats were homogenized in the RIPA Lysis buffer containing $50 \mathrm{mM}$ Tris (pH 7.4), $150 \mathrm{mM} \mathrm{NaCl}, 1 \%$ Triton X-100, $1 \%$ sodium deoxy cholate, $0.1 \%$ SDS overnight at $4^{\circ} \mathrm{C}$. The protein concentration 
A

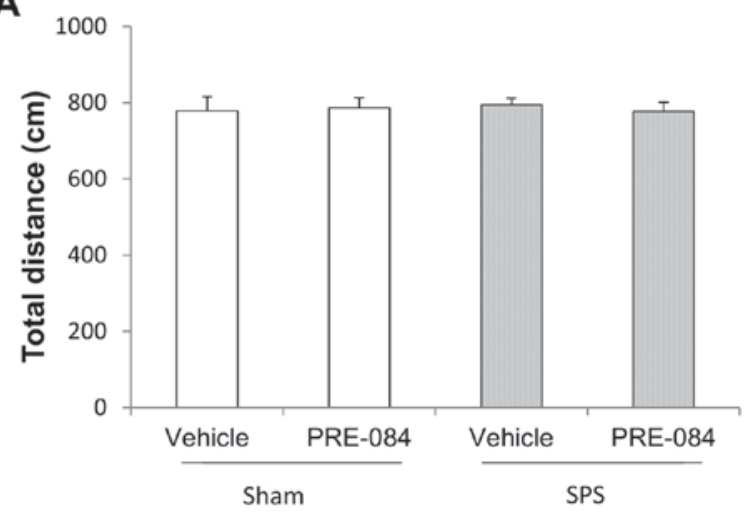

B
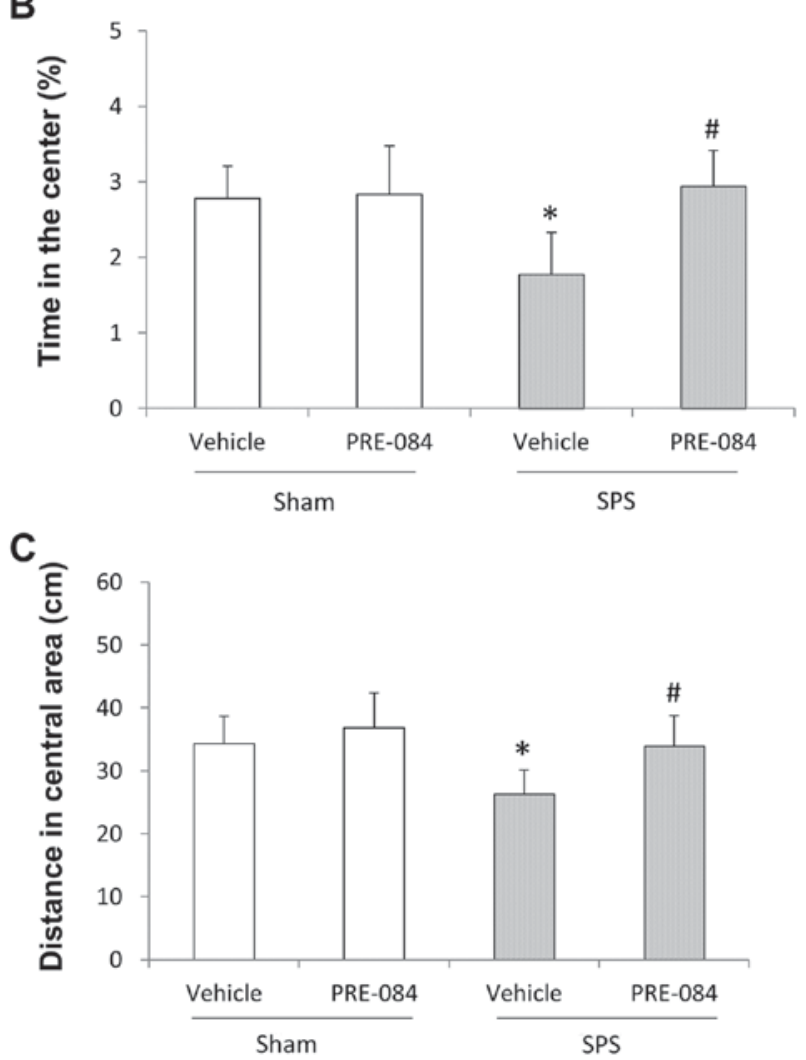

Figure 1. Effects of Sig-1R activation on behavior in the open field test. Total distance (A). Time in the center (B), and distance in the central area (C). Data are expressed as mean \pm SD with 12 rats per group. Statistically significant difference between two groups has been marked on the diagram. ${ }^{*} \mathrm{P}<0.05$ vs. Sham + Vehicle group, ${ }^{\#} \mathrm{P}<0.05$ vs. SPS + Vehicle group.

was determined after centrifugation and the samples were denatured by boiling for $5 \mathrm{~min}$. The protein fraction (30 $\mu \mathrm{g} / \mathrm{lane})$ extracted from each sample was separated by $10 \%(\mathrm{w} / \mathrm{v})$ gradient SDS-polyacrylamide gel electrophoresis (PAGE) and transferred to a $0.45 \mu \mathrm{m}$ PVDF membrane (Millipore Corp., Billerica, MA, USA). Membrane were blocked with $5 \%$ fetal calf serum in $0.05 \%$ Tween-20-containing TBST at room temperature for $2 \mathrm{~h}$ and incubated with rabbit polyclonal antibody against BDNF (Abcam; 1:1,000), rabbit polyclonal antibody against CREB and p-CREB (Abcam; 1:1,000) and rabbit polyclonal antibody against NR2A (Abcam; 1:1,000) overnight at $4^{\circ} \mathrm{C}$. After washed three times with TBST, the membrane was incubated with horseradish peroxidase-linked secondary antibodies against rabbit (ZSBio, Beijing, China;
A
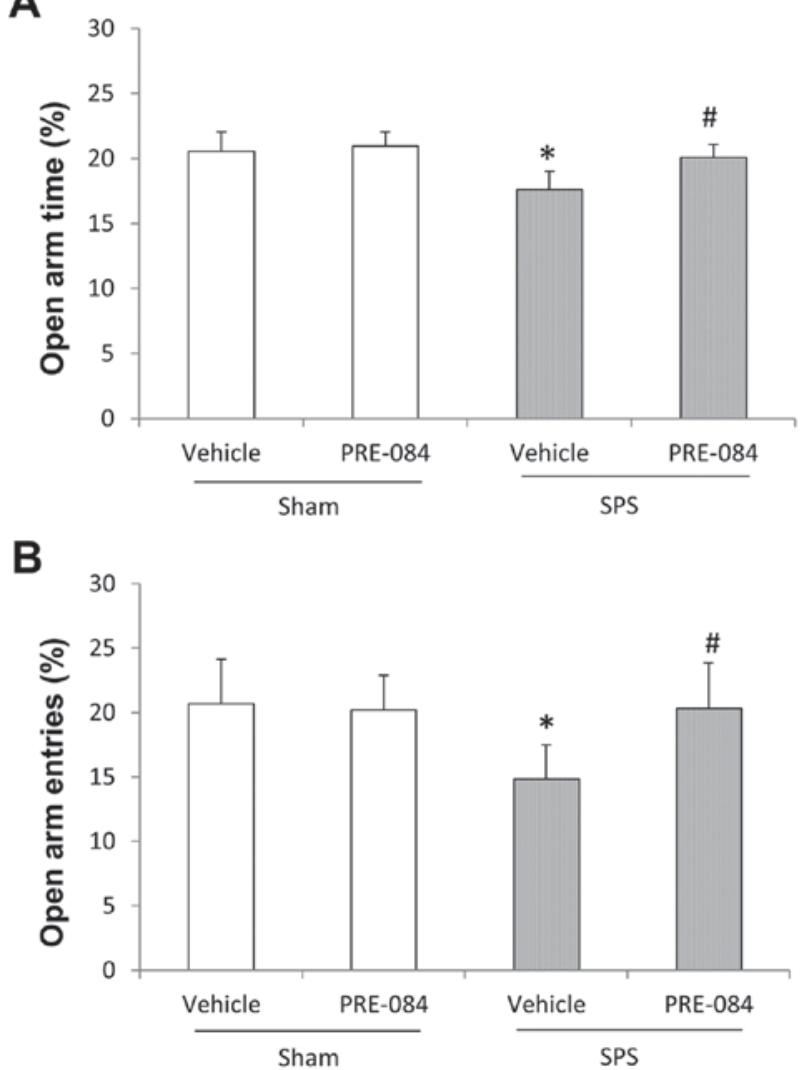

Figure 2. Effects of Sig-1R activation on behavior in the elevated plus maze test. Comparisons of the percentage of time spent in the open arms (A) and the percentage of open arms entries (B) are shown. Data are expressed as expressed as mean \pm SD with 12 rats per group. Statistically significant difference between two groups has been marked on the diagram. ${ }^{*} \mathrm{P}<0.05$ vs. Sham + Vehicle group, ${ }^{\#} \mathrm{P}<0.05$ vs. SPS + Vehicle group.

1:5,000) for another $1 \mathrm{~h}$ at $37^{\circ} \mathrm{C}$. After washed three times with TBST, the PVDF membrane were developed using enhanced chemiluminescence (ECL; Uscnlife, China). The intensity of peroxydase activity was qualified using the ImageJ software (National Institutes of Health, Bethesda, MD, USA).

Statistical analysis. Data are presented as mean \pm standard deviation of the mean (SD) and analyzed with SPSS software (version 22.0, IBM SPSS, Armonk, NY, USA). One-way or two-way analysis of variance (ANOVA) with post hoc Tukey's test was used to determine statistical significance. $\mathrm{P}<0.05$ was considered to indicate a statistically significant difference.

\section{Results}

The effects of Sig-1R activation on the impairment of anxiety behavior induced by PTSD in the OFT and EPM test. Open field test was performed to examine both locomotion and anxiety-like behavior. Gross motoric behavior was not affected by both SPS exposure and PRE-084 administration, because no significant differences were found in the total distance among the four groups ( $\mathrm{P}>0.05$; Fig. 1A).

Two-way ANOVA showed significant effects of Regent $[\mathrm{F}(1,20)=7.987, \mathrm{P}<0.05]$, interaction between Treatment and Regent $[\mathrm{F}(1,20)=6.601, \mathrm{P}<0.05]$, but not Treatment $[F(1,20)=4.224, P>0.05]$ in time in the center (Fig. 1B) 

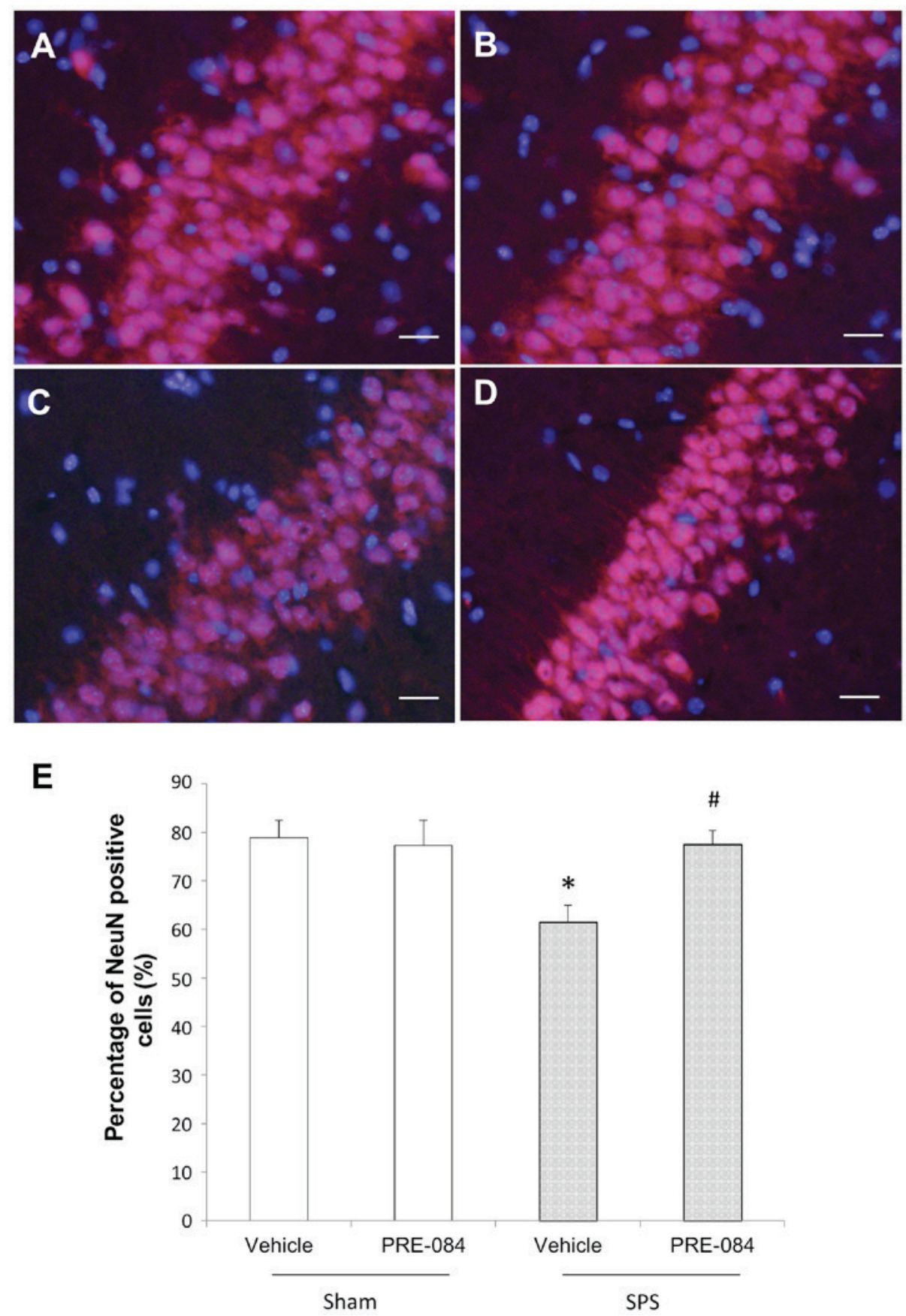

Figure 3. Representative images showing the immunoreactivity of NeuN (A-D) in rat hippocampus. Antibodies specific to NeuN were used. Compared to control (A), NeuN immunoreactivity decreased after SPS pradigm (C). PRE-084 increased SPS-induced immunoreactivity of NeuN (D). Sham + Vehicle group (A), Sham + PRE-084 group (B), SPS + Vehiclegroup (C), SPS + PRE-084 group (D), graphic representation of the percentage of NeuN positive cells. Quantitative analysis of the mean optical density (E). All cells were counterstained by DAPI (blue), Scale bar, $50 \mathrm{~mm}$; The relative levels are presented as the mean $\pm \mathrm{SD}, \mathrm{n}=6$ per group. ${ }^{*} \mathrm{P}<0.05$ vs. Sham + Vehicle group, ${ }^{\prime} \mathrm{P}<0.05$ vs. $\mathrm{SPS}+$ Vehicle group.

and significant effects of Regent $(\mathrm{F}(1,20)=7.253, \mathrm{P}<0.05)$, Treatment $(\mathrm{F}(1,20)=8.236, \mathrm{P}<0.05)$, but not interaction between Treatment and Regent $(F(1,20)=1.873, P>0.05)$ in distance in the center.

Tukey's post hoc analysis revealed that the time and distance in the center were significantly reduced in SPS rats compared to the control group $(\mathrm{P}<0.05$, respectively). After PRE-084 administration, the time in the center in the SPS + PRE-084 group was higher than that in the SPS + Vehicle group $(\mathrm{P}<0.05)$, and the distance in the center in the SPS + PRE-084 group was more than that in the SPS + Vehicle group $(\mathrm{P}<0.05)$, suggesting that anxiety behavior could be alleviated by Sig-1R activation.

In order to further testify this result, the elevated plus maze test was applied one hour after OFT. Two-way ANOVA showed significant effects of Regent $(\mathrm{F}(1,20)=7.793, \mathrm{P}<0.05)$, Treatment $[F(1,20)=13.387, \mathrm{P}<0.05]$, but not interaction between Treatment and Regent $[F(1,20)=3.914, P>0.05]$ on the percentage of time spent in the open arms (Fig. 2A) and Treatment $[\mathrm{F}(1,20)=5.009$, $\mathrm{P}<0.05]$, interaction between Treatment and Regent $[F(1,20)=5.615, P<0.05]$, but not Regent $[F(1,20)=3.899, P>0.05]$ on the percentage of open arm entries (Fig. 2B). 
A

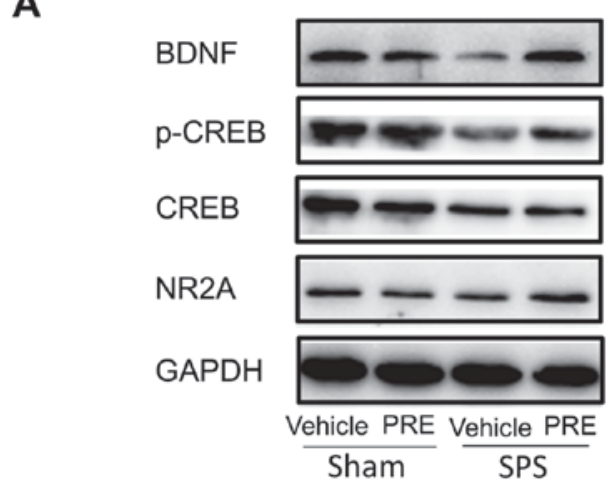

C

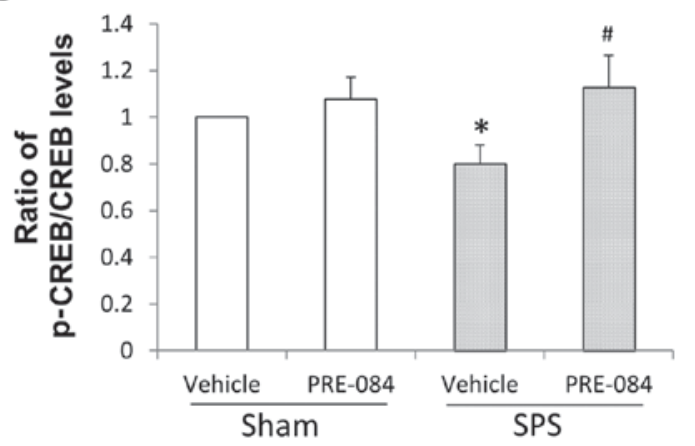

B

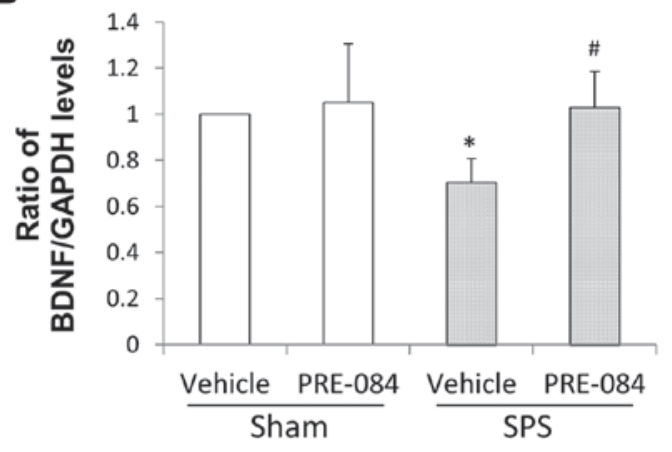

D

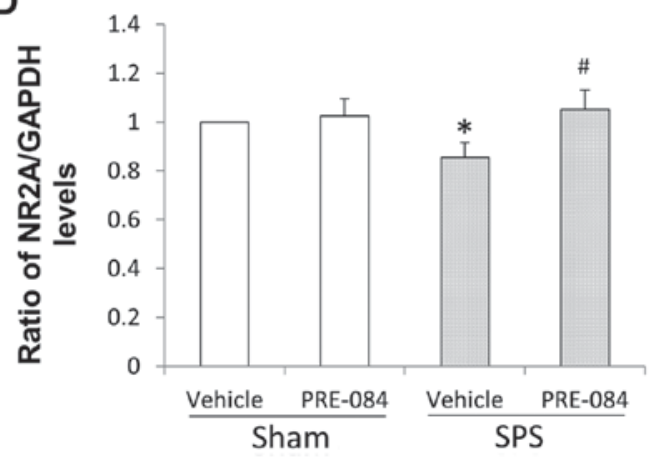

Figure 4. Western blot analysis demonstrating the expression of BDNF, CREB, p-CREB and NR2A in the hippocampus. Western blots for each protein are presented with GAPDH as a loading control (A), The relative levels are presented as the mean \pm SD (B-D), $n=6$ per group. * $<<0.05$ vs. Sham + Vehicle group, ${ }^{\#} \mathrm{P}<0.05$ vs. SPS + Vehicle group.

Tukey's post hoc analysis revealed that the time spent in the open arms and the frequency of entry into the open arms was significantly reduced in SPS rats compared to the control group. Meanwhile, PRE-084 treatment significantly increased the percentage of open arm time and the percentage of open arm entries in the SPS + PRE-084 group compared with the SPS + Vehicle group $(\mathrm{P}<0.01$, respectively), indicating that systemic PRE-084 application alleviated the anxiety response as expected.

The effects of Sig-1R activation on the expression of NeuN in hippocampus of SPS rats. The hippocampus was treated with immunofluorescence staining, as shown in Fig. 3A-D. NeuN-immunopositive cells were red, most of which showed clear nuclear; NeuN expression was evaluated by mean optical densities. A significant downregulation was shown in the SPS group compared to the control group ( $\mathrm{P}<0.05$, Fig. $3 \mathrm{C})$. While, the immunoreactivity increased significantly after administration of PRE-084 ( $\mathrm{P}<0.05$ vs. SPS, Fig. 3D). This result suggested that treatment with PRE-084 could reduce neuronal cell loss in the hippocampi of SPS rats.

The effects of Sig-1R activation on the NR2A-CREB-BDNF pathway in SPS rats. In order to investigate the possible mechanism of Sig-1R activation on reducing loss of neurons, we detected the expressions of BDNF in the hippocampus through western blotting. We found that the OD value of BDNF bands had a significant reduction in SPS group compared to the control group $(\mathrm{P}<0.05$, Fig. 4B). But the results are reversed by PRE-084 administration. To further examine the effect of Sig-1R activation on BDNF signaling, we detected CREB, p-CREB and the upstream event, NR2A with western blotting. The ratio of $\mathrm{p}-\mathrm{CREB}$ over CREB (regulator of BDNF) decreased dramatically in SPS group, while the expression was upregulated by PRE-084 administration $(\mathrm{P}<0.05$ vs. SPS group; Fig. 4C). Furthermore, we found that the level of NR2A was reduced in SPS rats compared with control group, while PRE-084 administration reversed this effect (Fig. 4D). These results suggest that Sig-1R is involved in the NR2A-CREB-BDNF signaling pathway.

\section{Discussion}

The aim of the present study was to investigate the role of Sig-1R activation on the anxiety-like behavior in an animal model of PTSD. In order to do this, rats were exposed to single-prolonged stress paradigm and chronically treated with PRE-084, a selective agonist of Sig-1R, at the dose of $0.6 \mathrm{mg} / \mathrm{kg}$ by intraperitoneal injections. The effects of Sig-1R activation on reduction of anxiety-like behaviors in SPS rats were verified by OFT and EPM test. Furthermore, the expression of BDNF, phospho-CREB and NR2A were detected to investigate the possible mechanism between Sig-1R activation and its anti-anxiety effects in PTSD rats. The reduction of expression level in BDNF, the ratio of p-CREB over CREB and the level of NR2A in hippocampi of SPS rats can be reversed by PRE-084 treatment.

An appropriate animal model will facilitate our understanding of the mechanism in effective treatment of PTSD. SPS paradigm was proposed as a rat model of PTSD, which 
consists of three different stress procedures: restraint for $2 \mathrm{~h}$, forced swim for $20 \mathrm{~min}$, and ether anesthesia (26,27,29-31). As such, SPS has been extensively developed and employed in the investigation of PTSD. In the present study, we found that SPS paradigms resulted in anxiety-like behaviors through OFT and EPM tests, consistent with our and other previous study. Furthermore, we observed the behaviors and changes of hippocampal neurons in SPS rats. We observed that SPS processes significantly decreased the number of neurons in hippocampus and resulted in the anxiety-like disorder, which could be ameliorated by chronic treatment of PRE-084, one selective agonists of Sig-1R, at a proper dose. An improvement in anxiety behaviors was induced by PRE-084 at the dose of $0.6 \mathrm{mg} / \mathrm{kg}$ after 7 days of treatment. These results suggest that the activation of Sig-1R can ameliorate anxiety like behavior disorders resulting from SPS by its neuroprotection effect.

In order to investigate the possible mechanism of anti-anxiety like effect of Sig-1R activation, we detected the expressions of BDNF, an activity-dependent growth factor and controlled by multiple signaling pathways. BDNF is widely expressed in the central nervous system and plays an important role in synaptic regulation, learning and memory, and neuroprotection. It is involved in pathological conditions of various neurodegenerative diseases, such as Huntington's disease and Alzheimer's disease (32-34). A reduction in BDNF secretion may lead to changes in volume of certain brain areas, such as hippocampus, amygdala and neocortex for its affection in dendritic and axonal growth (35-37). It has been reported that hippocampal volume is consistently reduced in some illness like depression and multiple sclerosis, and this symptom is associated with BDNF. Although the signaling pathway correlated with BDNF was reported to likely be a contributing factor in PTSD, as a matter of fact, changes in BDNF signaling may increase the risk of developing stress related psychopathology. Some studies suggested that sustained concentration of $\mathrm{p}-\mathrm{CREB}$, a transcriptional regulator of BDNF, could elevate the concentration of BDNF in neurodegenerative disease (38-40). It has been shown that CREB implicates in mood disorders and a wide variety of standard antidepressant treatments increase hippocampal CREB activity (41,42). Some studies suggested that CREB play a role in anxiety disorders, because CREB knockout mice display increase in anxiety-like behaviors and anxiety-like behaviors can be modified by altering CREB function or expression (43-45). In the present study, we found that the BDNF expression in rat's hippocampus was decreased by SPS Paradigms, accompanied by a decrease of the ratio of p-CREB over CREB. As we proposed, the ratio of p-CREB over CREB was increased, accompanied by an increased transportation of BDNF after chronic treatment with PRE-084 at the proper dose. These results suggested that Sig-1R activation may rescue the loss of neurons resulted from SPS and thus ameliorate the anxiety-like behaviors through increasing BDNF levels, possibly contributing to the enhancement of CREB phosphorylation in hippocampi of SPS rats.

Given that little is known about the pathophysiology of PTSD or the therapeutic mechanism of drugs used in the treatment of PTSD, we further investigated one of the factors correlated with both CREB and Sig-1R. Some studies have shown that Sig-1R can modulate multiple intracellular pathways and signaling cascades including $\mathrm{Ca}^{2+}$ ions, which played a pivotal role in cell death and neurodegenerative disease. The neuroprotection effect of Sig-1R activation could be through preventing detrimental elevations of intracellular $\mathrm{Ca}^{2+}$ by NMDA receptors $(46,47)$. Therefore, we detected the level of NR2A, a subunit of NMDA receptor and one of the regulators of CREB, which was implicated in learning and memory processes and also in cell death associated with neurological disorders, so as to verify if it could be modulated by Sig-1R. We found that PRE-084 induced NR2A up-regulation in hippocampi of SPS rats, in parallel with the improvement of anxiety-like behavior by Sig-1R activation. As mentioned above, PRE-084 was found to enhance the expression of BDNF and the ratio of $\mathrm{p}-\mathrm{CREB}$ over CREB, as measured by western blot. Lastly, although our study implicated the hippocampal NMDA-CREB-BDNF pathway as one of the factors in the enhancement of anxiety-like behavior by Sig-1R activation, other downstream or/and pathways may also be involved. This possible mechanism of action of Sig-1R activation awaits further investigation.

In summary, the results of the present study demonstrate that Sig-1R activation ameliorate the anxiety-like behavior in SPS rats. The expressions of BDNF, p-CREB and NR2A expressions in hippocampi of SPS rats were significantly increased by chronic treatment with PRE-084. The NR2A-CREB-BDNF pathway is possibly involved in the regulation of Sig-1R activation on anti-anxiety behavior in PTSD rats. Although the precise mechanism of this result is unknown, we suggested that NMDA-mediated signal transduction in the hippocampus may be involved in the pharmacology of Sig-1R activation on anti-anxiety effect in PTSD. Further studies will investigate both other neural mechanisms underlying Sig-1R agonists and the efficacy of Sig-1R agonists in the treatment of PTSD.

\section{Acknowledgements}

The present study was supported by the Natural Science Foundation of Liaoning Province, China (nos. 2015020459 and 201602825).

\section{References}

1. Parsons RG and Ressler KJ: Implications of memory modulation for post-traumatic stress and fear disorders. Nat Neurosci 16: 146-153, 2013.

2. Foley AM, Ammar ZM, Lee RH and Mitchell CS: Systematic review of the relationship between amyloid- $\beta$ levels and measures of transgenic mouse cognitive deficit in alzheimer's disease. J Alzheimers Dis 44: 787-795, 2015.

3. Vinters HV: Emerging concepts in alzheimer's disease. Annu Rev Pathol 10: 291-319, 2015.

4. Song JH, Yu JT and Tan L: Brain-derived neurotrophic factor in Alzheimer's disease: Risk, mechanisms and therapy. Mol Neurobiol 52: 1477-1493, 2015.

5. Ayton S, Lei P and Bush AI: Biometals and their therapeutic implications in Alzheimer's disease. Neurotherapeutics 12: 109-120, 2015.

6. Henriques AG, Oliveira JM, Carvalho LP and da Cruz E Silva OAB: Abeta influences cytoskeletal signaling cascades with consequences to Alzheimer's disease. Mol Neurobiol 52: 1391-1407, 2015

7. Bossini L, Tavanti M, Lombardelli A, Calossi S, Polizzotto NR, Galli R, Vatti G, Pieraccini F and Castrogiovanni P: Changes in hippocampal volume in patients with post-traumatic stress disorder after sertraline treatment. J Clin Psychopharmacol 27: 233-235, 2007. 
8. Alonso G, Phan V, Guillemain I, Saunier M, Legrand A, Anoal $\mathrm{M}$ and Maurice T: Immunocytochemical localization of the sigma(1) receptor in the adult rat central nervous system. Neuroscience 97: 155-170, 2000.

9. Skrzycki M and Czeczot H: Altered expression level of Sigma1 receptor gene in human colorectal cancer. J Recept Signal Transduct Res 33: 313-318, 2013.

10. Hayashi T and Su TP: Intracellular dynamics of sigma-1 receptors (sigma(1) binding sites) in NG108-15 cells. J Pharmacol Exp Ther 306: 726-733, 2003 .

11. Kourrich S, Hayashi T, Chuang JY, Tsai SY, Su TP and Bonci A Dynamic interaction between sigma-1 receptor and Kv1.2 shapes neuronal and behavioral responses to cocaine. Cell 152: 236-247, 2013.

12. Kimura Y, Fujita Y, Shibata K, Mori M and Yamashita T: Sigma-1 receptor enhances neurite elongation of cerebellar granule neurons via TrkB signaling. PLoS One 8: e75760, 2013.

13. Kinoshita M, Matsuoka Y, Suzuki T, Mirrielees J and Yang J: Sigma-1 receptor alters the kinetics of Kv1.3 voltage gated potassium channels but not the sensitivity to receptor ligands. Brain Res 1452: 1-9, 2012.

14. Martina M, Turcotte ME, Halman S and Bergeron R: The sigma-1 receptor modulates NMDA receptor synaptic transmission and plasticity via SK channels in rat hippocampus. J Physiol 578: 143-157, 2007.

15. Yagasaki Y, Numakawa T, Kumamaru E, Hayashi T, Su TP and Kunugi H: Chronic antidepressants potentiate via sigma-1 receptors the brain-derived neurotrophic factor-induced signaling for glutamate release. J Biol Chem 281: 12941-12949, 2006.

16. Hayashi T and Su TP: Sigma-1 receptor chaperones at the ER-mitochondrion interface regulate $\mathrm{Ca}(2+)$ signaling and cell survival. Cell 131: 596-610, 2007.

17. Prause J, Goswami A, Katona I, Roos A, Schnizler M, Bushuven E, Dreier A, Buchkremer S, Johann S, Beyer C, et al Altered localization, abnormal modification and loss of function of Sigma receptor-1 in amyotrophic lateral sclerosis. Hum Mol Genet 22: 1581-1600, 2013.

18. Ji LL, Peng JB, Fu CH, Cao D, Li D, Tong L and Wang ZY: Activation of Sigma-1 receptor ameliorates anxiety-like behavior and cognitive impairments in a rat model of post-traumatic stress disorder. Behav Brain Res 311: 408-415, 2016.

19. Dai HB, Ji X, Zhu SH, Hu YM, Zhang LD, Miao XL, Ma RM, Duan ML and Li WY: Hydrogen sulphide and mild hypothermia activate the CREB signaling pathway and prevent ischemia-reperfusion injury. BMC Anesthesiol 15: 119, 2015.

20. Leaderbrand K, Corcoran KA and Radulovic J: Co-activation of NR2A and NR2B subunits induces resistance to fear extinction. Neurobiol Learn Mem 113: 35-40, 2014

21. Rauner C and Köhr G: Triheteromeric NR1/NR2A/NR2B receptors constitute the major $\mathrm{N}$-methyl-D-aspartate receptor population in adult hippocampal synapses. J Biol Chem 286: 7558-7566, 2011

22. Liu Y, Wong TP, Aarts M, Rooyakkers A, Liu L, Lai TW, Wu DC, Lu J, Tymianski M, Craig AM and Wang YT: NMDA receptor subunits have differential roles in mediating excitotoxic neurona death both in vitro and in vivo. J Neurosci 27: 2846-2857, 2007.

23. Nalivaeva NN, Belyaev ND, Kerridge C and Turner AJ: Amyloid-clearing proteins and their epigenetic regulation as a therapeutic target in Alzheimer's disease. Front Aging Neurosci 6: 235, 2014

24. Hetman M and Kharebava G: Survival signaling pathways activated by NMDA receptors. Curr Top Med Chem 6: 787-799, 2006.

25. Pandey SC, Roy A and Zhang H: The decreased phosphorylation of cyclic adenosine monophosphate (cAMP) response element binding (CREB) protein in the central amygdala acts as a molecular substrate for anxiety related to ethanol withdrawal in rats. Alcohol Clin Exp Res 27: 396-409, 2003.

26. Takahashi T, Morinobu S, Iwamoto Y and Yamawaki S: Effect of paroxetine on enhanced contextual fear induced by single prolonged stress in rats. Psychopharmacology (Berl) 189: $165-173,2006$.

27. Liberzon I, Krstov M and Young EA: Stress-restress: Effects on ACTH and fast feedback. Psychoneuroendocrinology 22 443-453, 1997.

28. Ji LL, Tong L, Xu BK, Fu CH, Shu W, Peng JB and Wang ZY: Intra-hippocampal administration of ZIP alleviates depressive and anxiety-like responses in an animal model of posttraumatic stress disorder. Behav Brain Funct 10: 28, 2014.
29. Liberzon I, López JF, Flagel SB, Vázquez DM and Young EA: Differential regulation of hippocampal glucocorticoid receptors mRNA and fast feedback: Relevance to post-traumatic stress disorder. J Neuroendocrinol 11: 11-17, 1999.

30. Khan S and Liberzon I: Topiramate attenuates exaggerated acoustic startle in an animal model of PTSD. Psychopharmacology (Berl) 172: 225-229, 2004

31. Imanaka A, Morinobu S, Toki S and Yamawaki S: Importance of early environment in the development of post-traumatic stress disorder-like behaviors. Behav Brain Res 173: 129-137, 2006.

32. Ferrer I, Goutan E, Marin C, Rey MJ and Ribalta T: Brain-derived neurotrophic factor in Huntington disease. Brain Res 866: 257-261, 2000

33. Hock C, Heese K, Hulette C, Rosenberg C and Otten U: Region-specific neurotrophin imbalances in Alzheimer disease: Decreased levels of brain-derived neurotrophic factor and increased levels of nerve growth factor in hippocampus and cortical areas. Arch Neurol 57: 846-851,2000.

34. Lu B, Nagappan G, Guan X, Nathan PJ and Wren P: BDNF-based synaptic repair as a disease-modifying strategy for neurodegenerative diseases. Nat Rev Neurosci 14: 401-416, 2013.

35. Pezawas L, Verchinski BA, Mattay VS, Callicott JH, Kolachana BS, Straub RE, Egan MF, Meyer-Lindenberg A and Weinberger DR: The brain-derived neurotrophic factor val66met polymorphism and variation in human cortical morphology. J Neurosci 24: 10099-10102, 2004.

36. Szeszko PR, Lipsky R, Mentschel C, Robinson D, Gunduz-Bruce H, Sevy S, Ashtari M, Napolitano B, Bilder RM, Kane JM, et al: Brain-derived neurotrophic factor val66met polymorphism and volume of the hippocampal formation. Mol Psychiatry 10: 631-636, 2005

37. Yang X, Liu P, Sun J, Wang G, Zeng F, Yuan K, Liu J, Dong M, von Deneen KM, Qin W and Tian J: Impact of brain-derived neurotrophic factor Val66Met polymorphism on cortical thickness and voxel-based morphometry in healthy Chinese young adults. PLoS One 7: e37777, 2012.

38. Xu Q,JiXF,Chi TY,Liu P,JinG,Gu SL andZouLB: Sigma 1 receptor activation regulates brain-derived neurotrophic factor through NR2A-CaMKIV-TORC1 pathway to rescue the impairment of learning and memory induced by brain ischaemia/reperfusion. Psychopharmacology (Berl) 232: 1779-1791, 2015.

39. Kokaia Z, Zhao Q, Kokaia M, Elmér E, Metsis M, Smith ML, Siesjö BK and Lindvall O: Regulation of brain-derived neurotrophic factor gene expression after transient middle cerebral artery occlusion with and without brain damage. Exp Neurol 136: 73-88, 1995.

40. Walton MR and Dragunow I: Is CREB a key to neuronal survival? Trends Neurosci 23: 48-53, 2000.

41. Carlezon WA Jr, Duman RS and Nestler EJ: The many faces of CREB. Trends Neurosci 28: 436-445, 2005.

42. Gourley SL, Wu FJ, Kiraly DD, Ploski JE, Kedves AT, Duman RS and Taylor JR: Regionally specific regulation of ERK MAP kinase in a model of antidepressant-sensitive chronic depression. Biol Psychiatry 63: 353-359, 2008.

43. Valverde O, Mantamadiotis T, Torrecilla M, Ugedo L, Pineda J, Bleckmann S, Gass P, Kretz O, Mitchell JM, Schütz G and Maldonado R: Modulation of anxiety-like behavior and morphine dependence in CREB-deficient mice. Neuropsychopharmacology 29: 1122-1133, 2004.

44. Barrot M, Wallace DL, Bolaños CA, Graham DL, Perrotti LI, Neve RL, Chambliss H, Yin JC and Nestler EJ: Regulation of anxiety and initiation of sexual behavior by CREB in the nucleus accumbens. Proc Natl Acad Sci USA 102: 8357-8362, 2005.

45. Wallace DL, Han MH, Graham DL, Green TA, Vialou V, Iñiguez $\mathrm{SD}$, Cao JL, Kirk A, Chakravarty S, Kumar A, et al: CREB regulation of nucleus accumbens excitability mediates social isolation-induced behavioral deficits. Nat Neurosci 12: 200-209, 2009

46. Ruscher K and Wieloch T: The involvement of the sigma-1 receptor in neurodegeneration and neurorestoration. J Pharmacol Sci 127: 30-35, 2015

47. Klette KL, Lin Y, Clapp LE, DeCoster MA, Moreton JE and Tortella FC: Neuroprotective sigma ligands attenuate NMDA and trans-ACPD-induced calcium signaling in rat primary neurons. Brain Res 756: 231-240, 1997. 